\title{
Physical Characteristics based MANET Routing Protocols for Campus Network
}

\author{
Puneet \\ Department of Electronics \\ Technology, Guru Nanak Dev \\ University, Amritsar
}

\author{
R. S. Sawhney \\ Department of Electronics \\ Technology, Guru Nanak Dev \\ University, Amritsar
}

\author{
Rajan Vohra \\ Department of Electronics \\ Technology, Guru Nanak Dev \\ University, Amritsar
}

\begin{abstract}
Mobile ad-hoc networks (MANETs) consist of mobile devices that rely on wireless media for bilateral communication. These can be set up anywhere and at any time because these require neither infrastructure nor central administration. This paper concentrates on the comparative performance evaluation of three reactive routing protocols Ad Hoc On-demand Distance Vector (AODV), Dynamic Source Routing (DSR) and Temporally Ordered Routing Algorithm (TORA) for the three different Physical Characteristics namely Direct Sequence, Frequency Hopping and Infra Red in campus network of Guru Nanak Dev University, Amritsar using OPNET MODELER version 14.5. The performance metrics used for our simulation are Wireless LAN delay, Retransmission Attempts, Throughput and Media Access Delay.
\end{abstract}

\section{General Terms}

Throughput, Media Access delay, Frequency Hopping, Infrared, Direct Sequence

\section{Keywords}

MANET, DSR, AODV, TORA, OPNET

\section{INTRODUCTION}

The telecommunication networks are growing exponentially since the last decade. With the advancement in the communication technology, the applications are increasing at a rapid rate. The biggest beneficiary of this advancement has been the user itself. For the ease of operation, access as well as mobility, the mobile ad-hoc networks have become the choice of the main stream users. Mobile ad hoc network is a collection of mobile devices forming a network without any supporting infrastructure such as base stations or access points [1]. Nodes in the network should be able to sense and discover with nearby nodes. Each node acts as router for sending and receiving the packets to/ from other node [2]. Due to the limited transmission range of wireless network interfaces, multiple network hops may be needed for one node to exchange data with another across the network. MANETS have found applications in various areas such as disaster sensing and mitigation, conferences, lectures, emergency situation in hospitals, meetings, trade fairs, crowd control, and battle fields [3].

\section{LITERATURE REVIEW}

Several researchers in the past have shown keen interest in modeling various routing protocols of MANET by means of different performance parameters and have used simulators of their choice for this purpose. Jahangir khan et al. [4] showed the combined performance of both AODV and DSR in intermediate nodes data transfer rate from source to destination based on the traffic load and delay. It suggested that if the MANET has to be setup for a small amount of time, then AODV can be a preferred choice due to low initial packet loss than DSR. Also, AODV has very good packet receiving ratio in comparison to DSR. Jahangir Khan [5] through his simulation results of wireless routing protocols under scalable mobile condition worked on the correctness and efficiency in QOS framework and analyzed that TORA delivers better QOS support and DSR routing protocol is not efficient for large networks with many mobile nodes in comparison to AODV in terms of traffic delay which increases overhead. The performance of the protocols for varying number of nodes was presented by Gurleen Kaur Walia [6] and resulted that DSR has poor delay performance than AODV. Our paper presents the simulation results of reactive routing protocols DSR, AODV and TORA for different performance parameters based on Direct Sequence, Frequency hopping and Infra Red physical characteristics.

\section{ROUTING PROTOCOLS OF MANET}

MANET is having three types of routing protocols that are (1) Proactive Protocols follow table driven approach that constantly updates list of destinations and routes [4] such as Destination sequence distance Vector (DSDV); (2) Hybrid routing protocols [6] such as Zone routing protocol (ZRP) is limiting the set of forwarding nodes and using the proactive routing algorithm for nearly placed nodes which usually forward data to far placed nodes. (3) Reactive Protocols or on demand protocols follow event driven approach that creates the routes whenever it required such as Ad hoc On demand Distance Vector Routing (AODV), Dynamic Source Routing (DSR), Temporally Ordered Routing Algorithm (TORA). The basic idea of on-demand routing protocols, is that a source node sends a route request[6] and makes routing decision based on received route reply, which may be sent by destination or intermediate node. On-demand routing has several advantages, such as simplicity, correctness and flexibility. 


\subsection{DSR}

DSR [7] is a reactive protocol as it doesn't use periodic advertisements. It computes the routes when necessary and then maintains them. Source routing is a routing technique in which the sender of a packet determines the complete sequence of nodes through which the packet has to pass; the sender explicitly lists this route in the packet's header, identifying each forward hop by the address of the next node to which transmit the packet on its way to the destination host. There are two significant stages in working of DSR: Route Discovery and Route Maintenance. DSR has a unique advantage by virtue of source routing. As the route is part of the packet itself, routing loops either short-lived or long-lived cannot be formed as they can be immediately detected and eliminated.

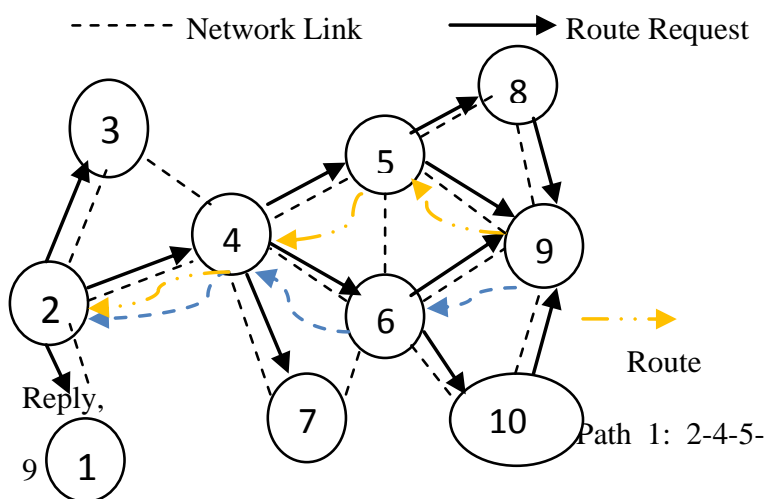

$-\rightarrow$ Route Reply, Path 2: 2-4-6-9

Figure 1: DSR routing protocol

\subsection{AODV}

AODV offers low network utilization and uses destination sequence number to ensure loop freedom. It is a reactive protocol implying that it requests a route when needed and it does not maintain routes for those nodes that do not actively participate in a communication. An important feature of AODV is that it uses a destination sequence number, which corresponds to a destination node that was requested by a routing sender node. If there are multiple routes from a request sender to a destination, the sender takes the route with a higher sequence number. This ensures that the ad hoc network protocol remains loop-free [8].

\subsection{TORA}

TORA [9] is a reactive routing protocol with some proactive enhancements where a link between nodes is established creating a Directed Acyclic Graph (DAG)[10] of the route from the source node to the destination. This protocol uses a link reversal model in route discovery. A route discovery query is broadcasted and propagated throughout the network until it reaches the destination or a node that has information about how to reach the destination. This protocol has an interesting property that it frequently chooses the most convenient route, rather than the shortest route. For all these attempts, TORA tries to minimize the routing management traffic overhead.

\section{SIMULATION MODEL}

The design goal is to evaluate the performance of AODV, DSR and TORA routing protocols of MANET for three different physical characteristics that are Direct Sequence, Frequency hopping and Infra red. The simulator that we are using is OPNET [11] (Optimized Network Engineering Tool) MODELER version 14.5. The Network Scenario created for simulation and evaluating the performance is given below:

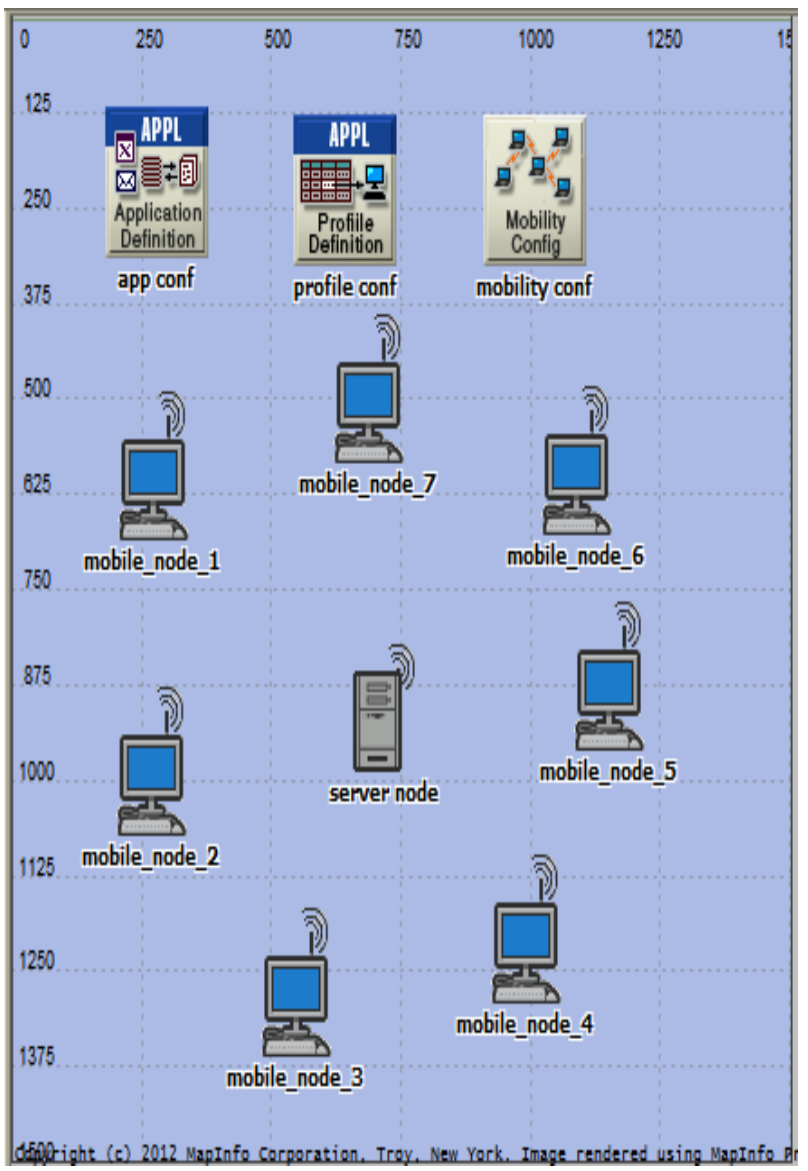

Figure 2: Network Scenario

In this study, the network of $1500 \mathrm{~m} \times 1500 \mathrm{~m}$ inside Guru Nanak Dev University, Amritsar, campus has been created with 7 WLAN mobile client nodes and one static WLAN server as reference prototype, but the same model can be implemented successfully for a larger network with large number of mobile nodes for the same kind of performance. This network has been configured for FTP (File Transfer Protocol) as an application at low load. Main characteristics of the scenarios maintained are depicted in the Table 1 . 
Table 1: Scenario Parameters

\begin{tabular}{|l|l|}
\hline \multicolumn{1}{|c|}{ Parameters } & \multicolumn{1}{c|}{ Values } \\
\hline Simulator & OPNET Modeler version 14.5 \\
\hline Protocols Studied & DSR, AODV, TORA \\
\hline Scenario Size & $1500 \mathrm{~m}$ x 1500 m \\
\hline Number of Nodes & 7 \\
\hline Data Rate & 2 Mbps \\
\hline Application Definition & FTP \\
\hline Physical Characteristics & $\begin{array}{l}\text { Direct sequence, Frequency } \\
\text { Hopping, Infra Red }\end{array}$ \\
\hline Node Movement Model & Random Waypoint Model \\
\hline Transmit Power (w) & 0.005 \\
\hline Buffer Size (bits) & 256000 \\
\hline
\end{tabular}

The scenarios were simulated for the three different physical characteristics that are direct sequence, frequency hopping and infra red for a fixed data rate $2 \mathrm{mbps}$ for the different routing protocols of MANET. Total 9 scenarios have been run for the simulation time of 30 minutes. 7 mobile nodes whose behavior has to be analyzed, when nodes move in the network with respect to time to determine the affecting features of each protocol. The Random Waypoint model has been selected to be used in all simulations presented in this document. Using Random Waypoint model, nodes keep moving until they arrive at a random destination calculated by the algorithm. To symbolize the mobile behavior of the nodes, the speed of the node is set to $10 \mathrm{~m} / \mathrm{s}$ with a pause time of $100 \mathrm{sec}$ to observe the network behavior.

FTP applications with low traffic load in line with the requirement for bandwidth utilization for data traffic analysis have been strategically selected. On the other hand, profile configuration is employed to create the user profiles whereas these profiles are specified on different nodes for generating the application traffic. For instance, an FTP profile is created in a profile configuration entity in order to support the FTP traffic, which is generated by an application configuration entity. The buffer size is set to 256,000 bits.

\section{PERFORMANCE PARAMETERS}

OPNET modeler supports different parameters for the measurement performance evaluation of the MANET network under different routing protocols. We evaluate four parameters in our study on overall network performance. These parameters are wireless LAN delay, retransmission attempts, throughput and media access delay.

\subsection{Wireless LAN delay}

The packet delay is the average time that packets take to transverse the network. This is the time from the generation of the packet by the sender up to their reception at the destination application layer and is expressed in seconds.

\subsection{Retransmission attempts}

Retransmission attempts are the resending of packets which have been either damaged or lost. Protocols which provide reliable communication over such networks use a combination of acknowledgments i.e. an explicit receipt from the destination of the data, retransmission of missing or damaged packets and checksums to provide that reliability.

\subsection{Throughput}

Throughput is the measurement of number of packets passing through the network in a unit of time. This metric show the total number of packets that have been successfully delivered to the destination nodes. The throughput is defined as the total amount of data a receiver receives from the sender divided by the time it takes for the receiver to get the last packet. It is expressed in bits per second or packets per second.

\subsection{Media access delay}

Media access delay is the time a node takes to access media (link) to start its transmission. Media access Delay includes queuing delays and the delays due to contentions and back offs.

\section{SIMULATION RESULTS AND ANALYSIS}

The results of simulation are studied and analyzed to get the performance of three routing protocols that is DSR, AODV and TORA for the different Physical characteristics of direct sequence, frequency hopping and infra red under simulation based on the parameters wireless LAN delay, retransmission attempts, throughput and media access delay. All the metric statistics are collected for all scenarios.

\subsection{Wireless LAN Delay}

Observing the characteristics in figures 3,4 and 5 , it is evident that AODV gives the lowest delay out of three protocols followed by TORA whose value initially increases and then decreases exponentially and finally becomes constant. The higher value of delay of TORA than AODV is due to its formation of temporary loops within the network, where the collisions are held by the transmitted routing packets. Consequently, the links to neighbor nodes are broken. Besides, in response to link failures, TORA sends more updated packets, whereas an acknowledgment of the re-transmitted update packet might not be received, resulting in a serious congestion of the network. DSR attains the higher value of delay throughout the period of simulation due to reactive approach, where the data packets keep on waiting in buffers until a route is discovered route to the destination. The results of three routing protocols are nearly same for the direct sequence and frequency hopping but when compared with the infra red the values of delay degrades to $.0006 \mathrm{sec}$ from $0.0010 \mathrm{sec}$ for AODV, 0.0012sec from $0.0020 \mathrm{sec}$ for TORA and $0.0022 \mathrm{sec}$ from $0.0026 \mathrm{sec}$ for DSR. The explanation for this observation is that as Wireless LAN 
delay is less, packets takes less time to transverse the network. Therefore AODV performs best in this case from other two protocols and the performance of infra red is better comparison for the other two physical characteristic.

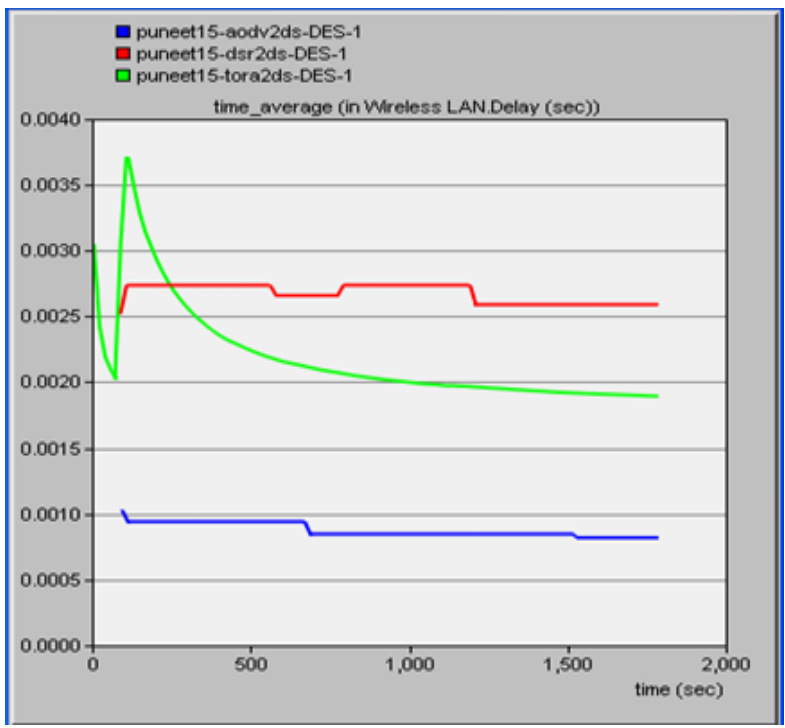

Figure 3: Delay in Direct sequence

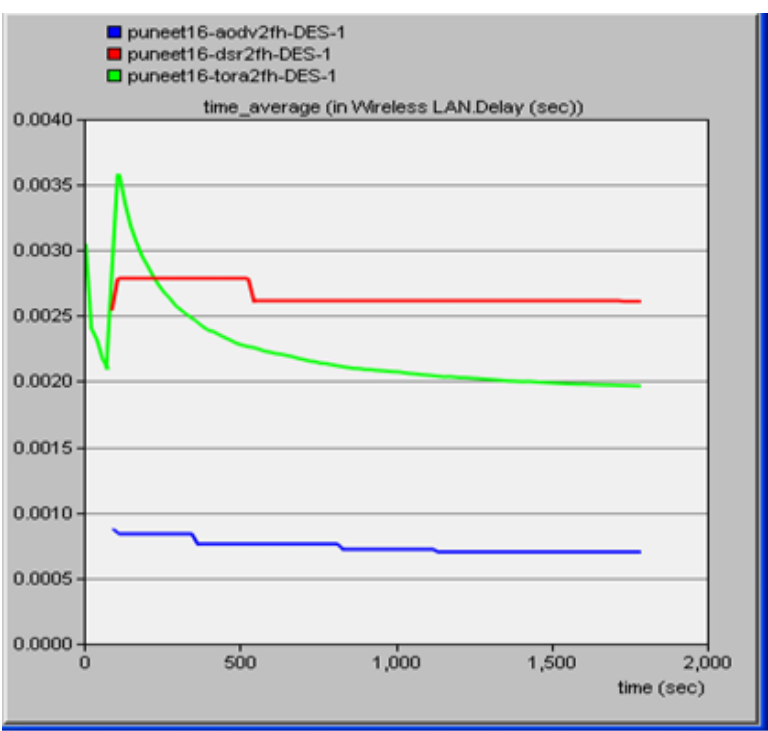

Figure 4: Delay in Frequency Hopping

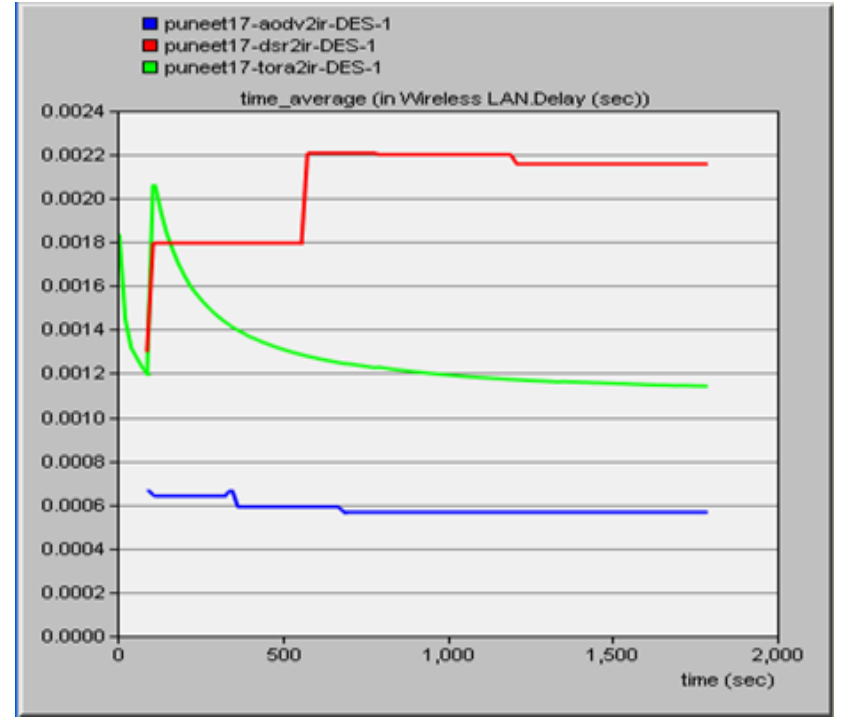

Figure 5: Delay in Infra Red

\subsection{Retransmission Attempts}

The figures 6,7 and 8 show the retransmission attempts of routing protocols in the different physical characteristics. Considering all the scenarios collectively, it is observed that AODV has least retransmission attempts and TORA suffers higher retransmission attempts. In case of direct sequence, there is no value of retransmission attempts for AODV, which means that all the packets are safely received by destination nodes and the value has been observed as 0.01 for Frequency hopping and 0.0075 for Infra red.

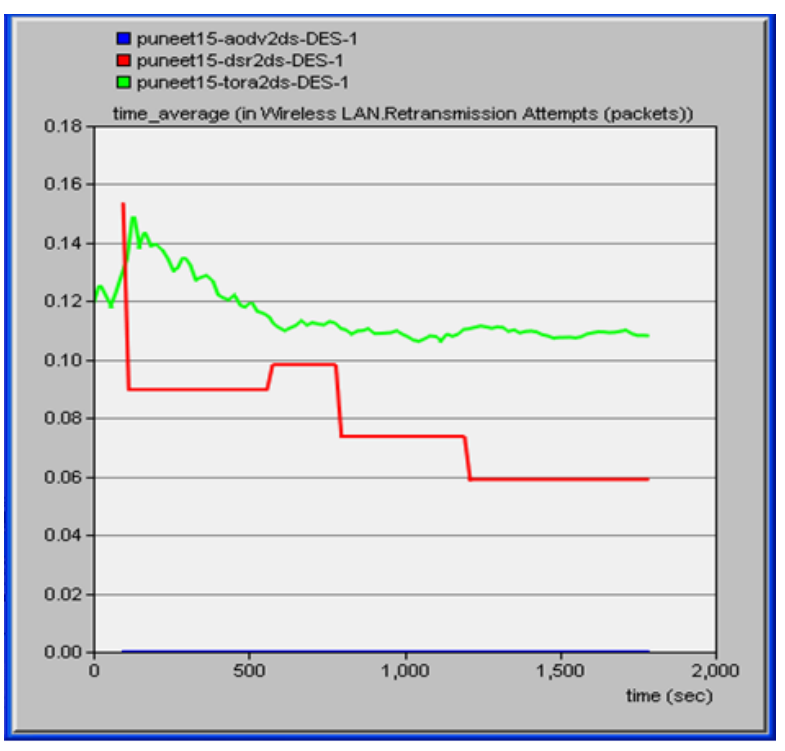

Figure 6: Retransmission Attempts in Direct Sequence 
In case of DSR, there is periodical reduction of retransmission attempts from 0.14 to 0.06 in direct sequence and averages at 0.09 in frequency hopping and 0.0075 in infra red which is nearly same as AODV. Retransmission attempts of TORA in frequency hopping is higher followed by direct sequence and then infra red. It is clear that more loss or damage of packets for the TORA routing protocols. Comparing all three approaches, infra red performs better than the other two physical characteristics.

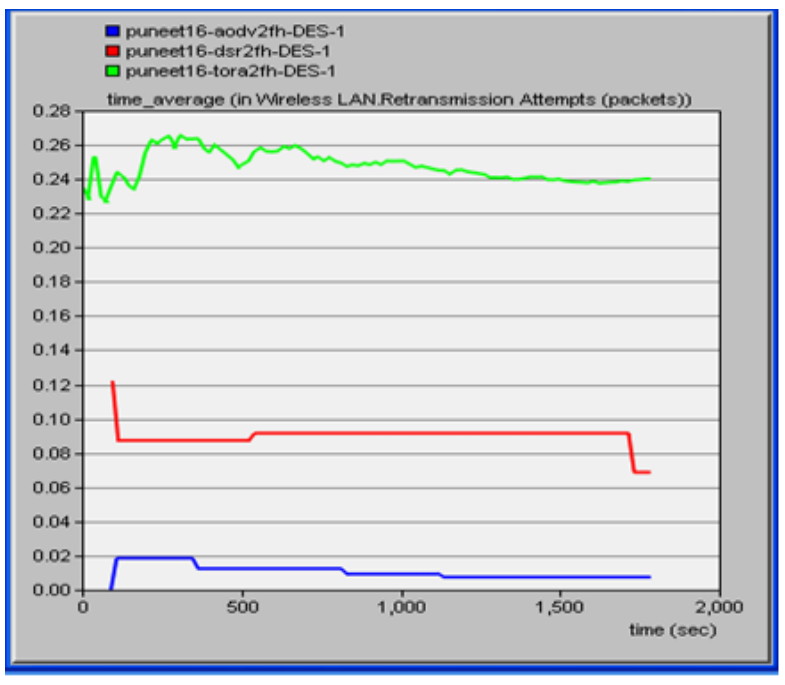

Figure 7: Retransmission Attempts in Frequency

\section{Hopping}

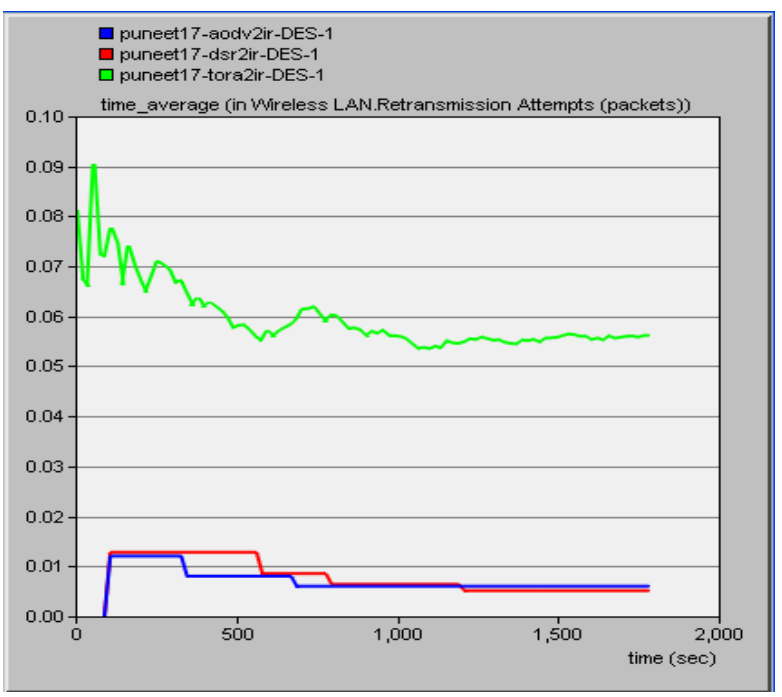

Figure 8: Retransmission Attempts in Infra Red

\subsection{Throughput}

The observation of the throughput, as noticed from the figures 9,10 and 11, explains that the TORA routing protocol far outperforms other protocols in all the three physical characteristics. Its peak value at $4000 \mathrm{bps}$ and then decreases exponentially, it attains a peak value at $4,000 \mathrm{bps}$ again before finally degrading to $1200 \mathrm{bps}$ with increase in time. The peak point of AODV is 3,000bps and that of DSR is $1,000 \mathrm{bps}$ and then both the characteristics exponentially decrease. The performance of all the three routing protocols is similar for all three physical characteristics. It means that data is received by the destination at the same amount of time for all the three physical characteristics. Since throughput is the ratio of the total amount of data that a receiver receives from the sender to the time it takes for the receiver to get the last packet. Comparing the DSR and AODV, the throughput of AODV performs better than the DSR routing protocol as already computed that delay of AODV is less than DSR.

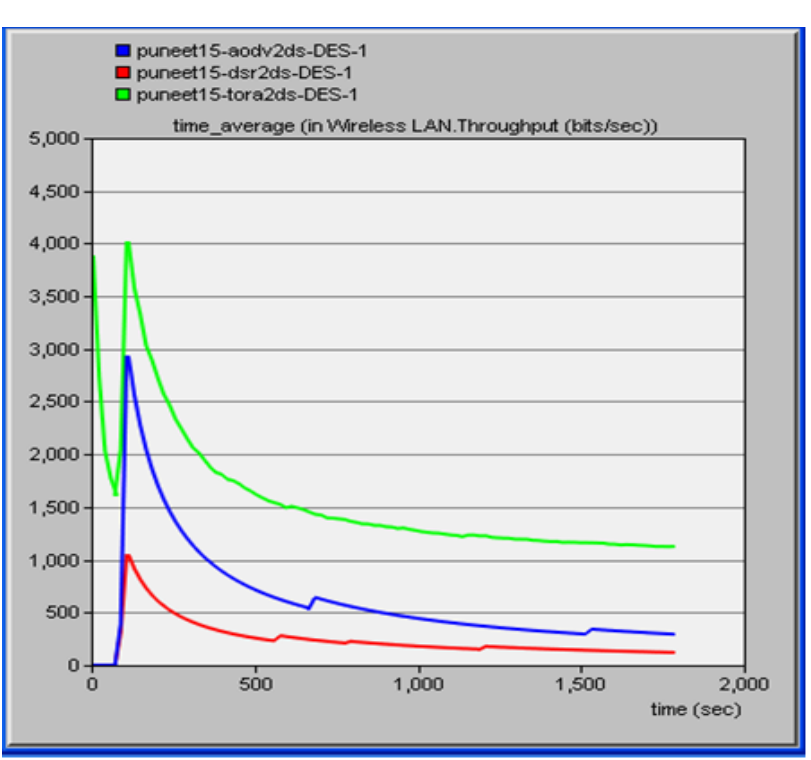

Figure 9: Throughput of Direct Sequence

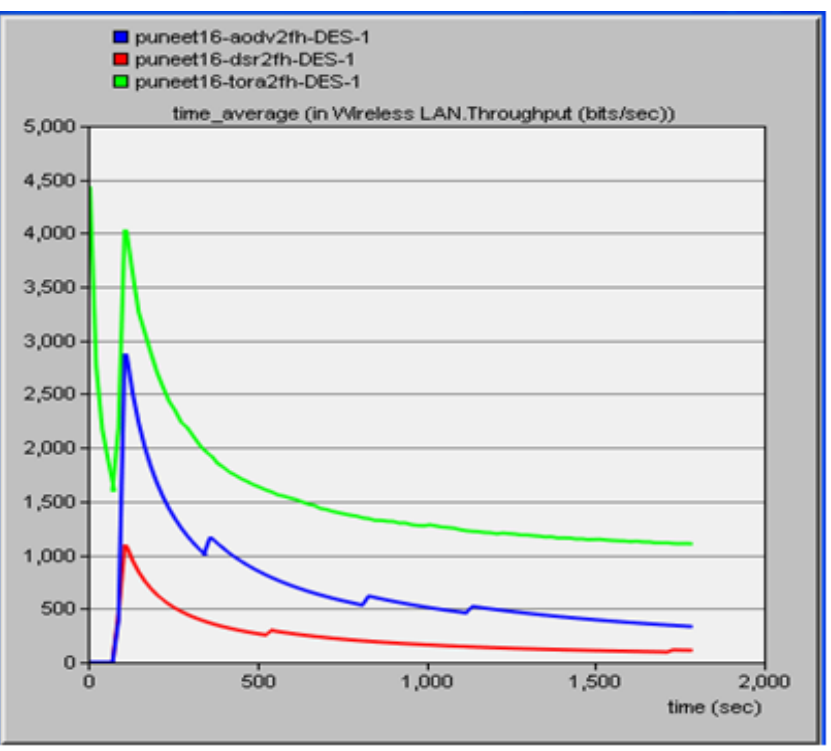

Figure 10: Throughput of frequency hopping 


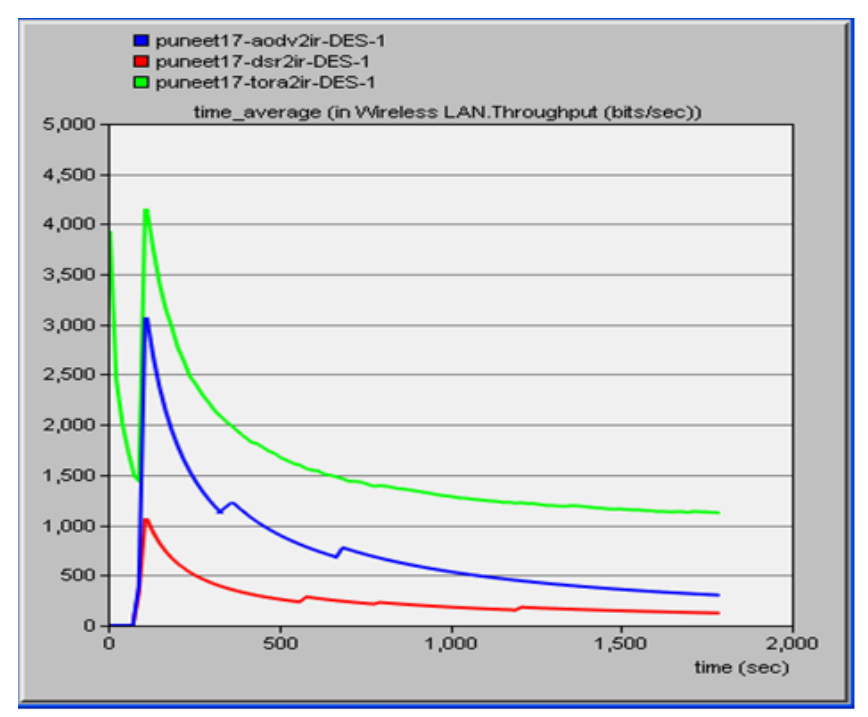

Figure 11: Throughput of Infra red

\subsection{Media Access Delay}

The figures 12, 13 and 14 show the media access delay of three routing protocols for three different physical characteristics. Considering all the scenarios collectively, it is observed that AODV performs better than DSR and TORA routing protocols for all three physical characteristics. AODV has value $0.009 \mathrm{sec}$ for both direct sequence and frequency hopping and degrades to $0.0006 \mathrm{sec}$ for infra red. Media access delay in case of TORA is initially high as it takes more time to access the link for the transmission of packets and then it gradually decreases. It suffers less media access delay for infra red as value degrades to $0.0014 \mathrm{sec}$ from $0.0023 \mathrm{sec}$.

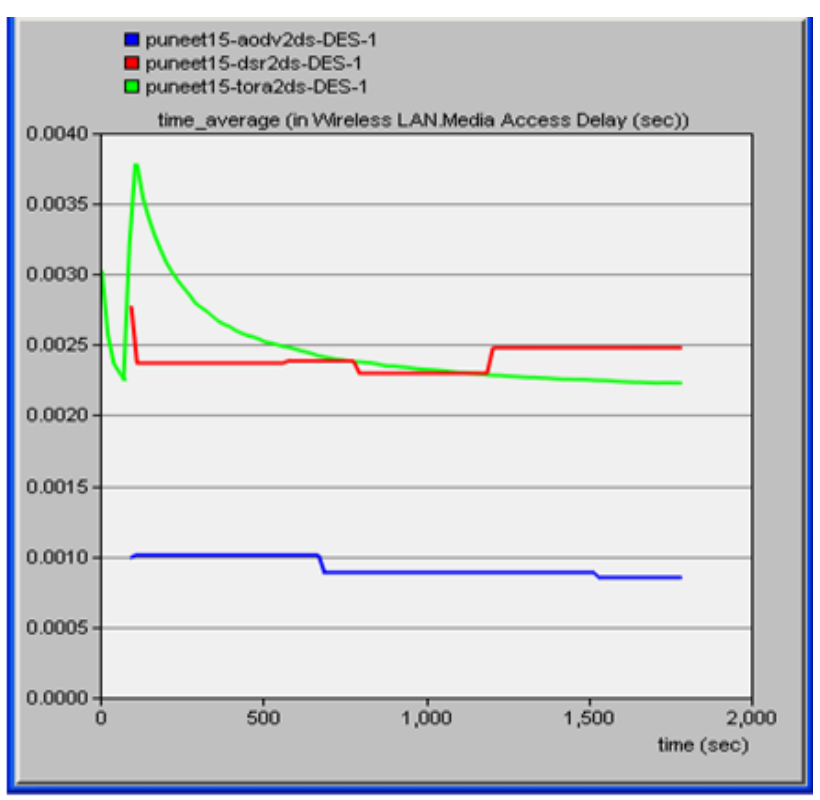

Figure 12: Media Access Delay of Direct

Sequence

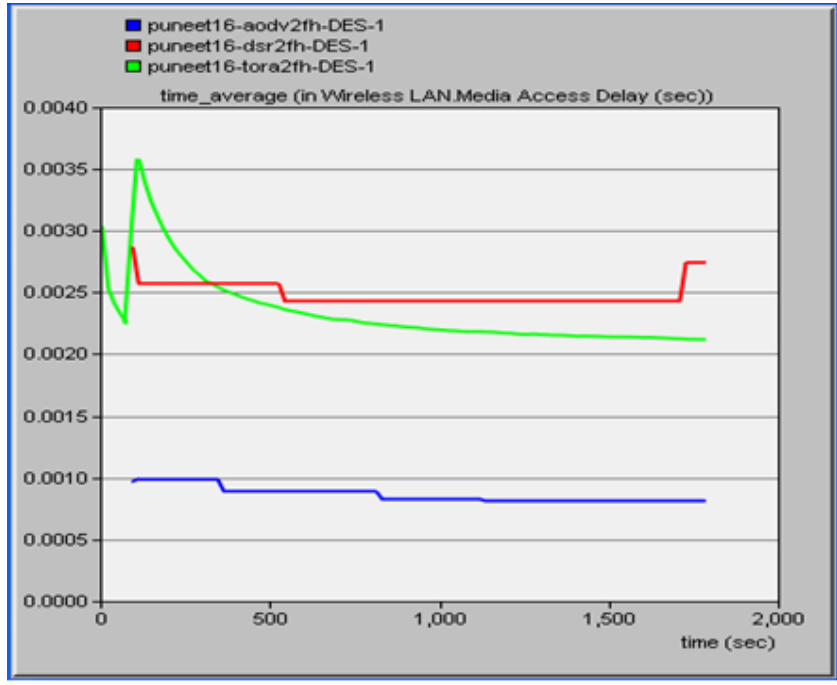

Figure 13: Media Access Delay of Frequency

Hopping

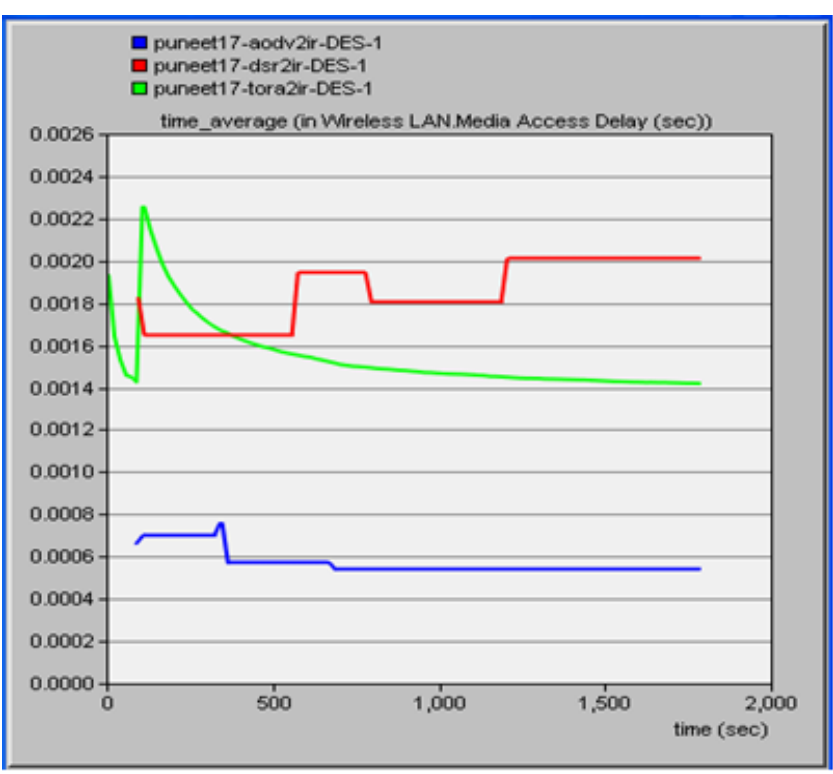

Figure 14: Media Access Delay of Infra Red

DSR remains at $0.0025 \mathrm{sec}$ for direct sequence and frequency hopping and degrades the value of delay to $0.0020 \mathrm{sec}$ in case of infra red. The performance of three routing protocols are consistent for direct sequence and frequency hopping and shows better results in infra red physical characteristics means protocols access the infra red media faster than the other physical characteristics.

\section{CONCLUSION}

Concluding the performance of the routing protocols based on physical characteristics, AODV performs best in case of Wireless LAN delay, retransmission attempts and media access delay due to frequent broadcasting of RREQ and route re-initialization messages to find an optimal fresh path, whereas TORA performs best in case of throughput as it does not engage in the route discovery again and again as it already have a DAG of all the nodes in the network. DSR has higher delay as DSR maintains a large 
route information table to store data transmission data and consume time during updating periodically with frequent changes occurring due to high mobility. In addition, the chance of using outdated or stale route information in forwarding packets is increased and probability of loss or damage of packets which causes the increase of retransmission attempts. On the other hand, infra red gives better results in parameters end to end delay, retransmission attempts and media access delay than direct sequence and frequency hopping while there is consistent performance of throughput in all the three physical characteristics. In the future scope, results are analyzed for the other physical characteristics and for the higher data rates using video conferencing application. The evaluation of more parameters for the routing protocols gives the more perfect results.

\section{ACKNOWLEDGMENT}

The authors would like to thank OPNET for modeling support through the OPNET Program tool.

\section{REFERENCES}

[1] Anil Kumar Verma, "Performance analysis of cluster based routing protocol in manets" MAY 2006

[2] Sandeep Kaur, "Simulation Analysis of AODV Routing Protocol of MANET using OPNET" IJCST Vol. 2, Issue 3, September 2011

[3] Razan Al-Ani, "Simulation and Performance Analysis Evaluation for Variant MANET Routing Protocols" International Journal of Advancements in Computing Technology, Volume 3, Number 1, February 2011

[4] Jahangir khan, Dr.syed Irfan Hyder, "Modeling and Simulation of Dynamic Intermediate Nodes and Performance Analysis in MANETS Reactive Routing protocols", International Journal of Grid and Distributed Computing Vol. 4, No. 1, March 2011
[5] Jahangir Khan, "Based on MANET the Correctness and Efficiency Evaluation of DSR, AODV and TORA Routing Protocols for Best QOS”, IJCA Special Issue on Mobile Ad-hoc Networks (MANETs), 2010

[6] Gurleen Kaur Walia, "Node Density based Performance Analysis of Two Reactive Routing Protocols in Mobile Ad-hoc Networks" International Journal of Computer Science and Information Technologies, Vol. 2 (3), 2011, 1229-1234

[7] Vahid Ayatollahi Tafti, Abolfazl Gandomi, "Performance of QoS Parameters in MANET Application Traffics in Large Scale Scenarios" World Academy of Science, Engineering and Technology 77 ,2011

[8] Geetha Jayakumar and Gopinath Ganapathy, "Performance comparison of Mobile Ad-hoc Network Routing Protocol," International Journal of Computer Science and Network Security (JJCSNS 2007), vol. VII, no. 11, pp. 77-84, November 2007

[9] G.Vijaya Kumar, "Current Research Work on Routing Protocols for MANET: A Literature Survey",International Journal on Computer Science and Engineering(IJCSE), Vol. 02, No. 03, 2010, 706713

[10] Park VD, "A highly adaptive distributed routing algorithm for mobile wireless networks", Proceedings of IEEE INFOCOM 1997, Volume 3:1405-1413

[11] OPNET MODELLER version 14.5, Modeler Wireless Suite.http://www.opnet.com/products/modeler 